\title{
Fosfomycin versus Nitrofurantoin for the Treatment of Lower UTI in Outpatients
}

\author{
Shraddha Sharma ${ }^{1}$ Pankaj Kumar Verma ${ }^{2}$ Vinita Rawat ${ }^{1} \quad$ Umesh Varshney $^{1}$ Rajesh Kumar Singh ${ }^{3}$ \\ ${ }^{1}$ Department of Microbiology, Govt. Medical College, Haldwani, \\ Nainital, Uttarakhand, India \\ ${ }^{2}$ Department of Surgery, Govt. Medical College, Haldwani, Nainital, \\ Uttarakhand, India \\ Address for correspondence Vinita Rawat, MD, Microbiology, \\ Department of Microbiology, Govt. Medical College, Haldwani, \\ Nainital, Uttarakhand, 263139, India \\ (e-mail: drvinitarawat31@rediffmail.com).
}

${ }^{3}$ Department of Community Medicine, Govt. Medical College, Haldwani, Nainital, Uttarakhand, India

J Lab Physicians 2021;13:118-122.

\begin{abstract}
Introduction Fosfomycin and nitrofurantoin are increasingly being prescribed in outpatients for the oral treatment of urinary tract infection (UTI). Although ample literature is available on the in vitro sensitivity pattern of fosfomycin and nitrofurantoin in UTI cases, clinical data are scant.

Methodology Voided midstream urine, collected from patients $\geq 16$ years of age of both genders with suspected sign and symptoms, was plated on cystine lactose electrolyte-deficient agar. Uropathogen was defined as an organism known to be associated with the signs and symptoms of UTI with $>10^{5}$ colony forming units/mL of urine. Antimicrobial susceptibility testing was determined by Kirby-Bauer disc diffusion method. Further, for fosfomycin, agar dilution method was also performed.

Results A total of 143 patients, 47 treated with fosfomycin and 96 with nitrofurantoin, were followed for clinical outcome. The most common isolated uropathogen was Escherichia coli. In vitro susceptibility rate of uropathogens against fosfomycin and nitrofurantoin was $99.3 \%$ and $81.2 \%$, respectively. Overall, the clinical cure rate with fosfomycin and nitrofurantoin treatment groups was $80.85 \%$ and $90.06 \%$ respectively (not statistically significant).

Keywords

- fosfomycin

- nitrofurantoin

- UTI

Conclusion Fosfomycin and nitrofurantoin showed good in vitro activity against uropathogens from lower UTI and can be used for empirical therapy in our area. Multiple confounding factors may have contributed to the discrepancy between in vitro susceptibility and clinical cure, which needs to be studied further.
\end{abstract}

\section{Introduction}

Urinary tract infection (UTI) is one of the most common community-acquired infections, and agents such as fluoroquinolone and co-trimoxazole are commonly used for the treatment of UTI. However, due to the increased prevalence of drug resistance globally, there has been a surge in the

published online May 30, 2021
DOI https://doi.org/

$10.1055 / \mathrm{s}-0041-1729141$ ISSN 0974-2727 efforts to identify newer treatment options or re-evaluate the existing agents for the treatment of UTIs, such as fosfomycin and nitrofurantoin. ${ }^{1-4}$ Fosfomycin and nitrofurantoin are now being increasingly prescribed in outpatients for the oral treatment of UTI. Although ample literature is available on the in vitro sensitivity pattern of fosfomycin and nitrofurantoin in UTI cases, clinical data are scant. (c) 2021. The Indian Association of Laboratory Physicians.

This is an open access article published by Thieme under the terms of the Creative Commons Attribution-NonDerivative-NonCommercial-License, permitting copying and reproduction so long as the original work is given appropriate credit. Contents may not be used for commercial purposes, or adapted, remixed, transformed or built upon. (https://creativecommons.org/licenses/by-nc-nd/4.0/).

Thieme Medical and Scientific Publishers Pvt. Ltd. A-12, 2nd Floor, Sector 2, Noida-201301 UP, India 


\section{Methodology}

Ethical clearance for this prospective study was obtained from the institutional ethic committee.

Clean voided midstream urine samples were collected from patients $\geq 16$ years of age of both genders with suspected signs and symptoms, along with clinical diagnosis of lower UTI.

Urine samples were plated on cystine lactose electrolyte-deficient (CLED) agar by standard loop method and were incubated overnight at $37^{\circ} \mathrm{C}$. Urinary pathogens were identified as per the standard protocol. ${ }^{5}$

We defined a uropathogen as an organism known to be associated with signs and symptoms of UTI with $>10^{5}$ colony forming units $/ \mathrm{mL}$ of urine. If a urine specimen grew > 2 organisms, it was considered as contaminated and was excluded from the study.

Antimicrobial susceptibility testing was determined by the Kirby-Bauer disc diffusion method and results were interpreted as per the guidelines of the Clinical and Laboratory Standards Institute (CLSI). ${ }^{5}$ The following antibiotics discs were used: fosfomycin $(200 \mu \mathrm{g} /$ disc), levofloxacin $(5 \mu \mathrm{g} / \mathrm{disc})$, nitrofurantoin $(300 \mu \mathrm{g} /$ disc), co-trimoxazole $(25 \mu \mathrm{g} / \mathrm{disc})$, and cephazolin $(30 \mu \mathrm{g} / \mathrm{disc})$. Fosfomycin trometamol resistance was also determined by the agar dilution method as per the CLSI guidelines $2017 .{ }^{6}$ Interpretative criteria from the CLSI for fosfomycin susceptibility are only available for Escherichia coli (E. coli) and Enterococcus faecalis. Therefore, results for other organisms were interpreted according to the criteria for E. coli.

A detailed questionnaire tracking the demographics, risk factors, and clinical information was recorded in a predesigned proforma at the time of the report delivery. This accompanying questionnaire enabled us to classify UTIs as complicated or uncomplicated. Patients were divided in two treatment groups: fosfomycin-treated group (FTG) and nitrofurantoin-treated group (NTG). Patients were asked for a repeat sample 10 to 12 days after the completion of treatment and to contact the study investigator in the absence of clinical improvement. Telephonic follow-ups were made with all the patients for 1 month to know the treatment outcome.

\section{Complicated UTI}

Male gender, pregnancy, history of UTI in the last 2 weeks, history of admission to the hospital in the last 30 days, presence of diabetes mellitus, obstructive uropathy, or the presence of a urinary catheter on sampling were considered as complicating factors. ${ }^{7}$ Acute uncomplicated cystitis (AUC)female nonpregnant patients without complicating factors, presenting with at least one urinary symptom (i.e., frequency, dysuria, hematuria, suprapubic pain, excluding fever, or vaginal symptoms) and a positive urine culture were assigned to the AUC group. ${ }^{7}$

We divided microbiological outcome into the following five categories:

1. Sterile urine-Microbiological cure

2. No follow-up sample available but clinical resolution was confirmed telephonically
3. No follow-up sample available for whom the persistence of symptoms was confirmed telephonically

4. Reinfection-development of UTI with a different organism within 30 days

5. Relapse-development of UTI with the same organism within 30 days

\section{Clinical Cure}

Category 1 and 2 were considered as clinical cure.

\section{Clinical Failure}

Category 3, 4, and 5 were considered as clinical failure.

\section{Statistical Analysis}

The data were entered in MS Excel and analysis was done using the software Epi Info version 7.2.3.1. Data were expressed in terms of frequency and percentage. Chi-squared test was used for the assessment of statistical significance of the difference between proportions. A $p$-value of $<0.05$ was considered statistically significant.

\section{Results}

A total of 294 symptomatic patients showed growth in urine culture during the study period (September 2017-December 2018). Out of these, only 164 patients could be followed up. From these 164 patients, 47 were treated with fosfomycin, 96 with nitrofurantoin, and 21 patients received additional antimicrobial agents along with fosfomycin or nitrofurantoin; hence, these 21 cases were excluded from our analysis. A total of 143 patients were included for analysis, and had 145 urinary pathogens. Baseline demographics and associated risk factors are depicted in - Table 1. Patients in fosfomycin and nitrofurantoin treatment groups were significantly varied on the basis of gender. The proportion of patients having obstructive uropathy and more than one risk factors was significantly different in the fosfomycin and nitrofurantoin treatment groups.

The most commonly isolated uropathogen was E. coli (81\%). The susceptibility rate of uropathogens against fosfomycin, nitrofurantoin, ciprofloxacin, cotrimoxazole, and cephalexin was $99.3 \%, 81.2 \%, 62 \%, 65 \%$, and $65 \%$, respectively. Intermediate sensitivity was considered as a resistant for analysis. These included six nitrofurantoin, two ciprofloxacin, one fosfomycin, and one cephalexin. Pathogen-wise antimicrobial resistance pattern is shown in - Table 2. In the present study, FTG received three doses of $3 \mathrm{~g}$ fosfomycin on alternate days, and NTG received five doses of $100 \mathrm{mg}$ nitrofurantoin every 12 hours. Overall, the clinical cure rate of fosfomycin and nitrofurantoin treatment groups was $80.85 \%$ and $90.06 \%$, respectively. However, this difference is not statistically significant. Category-wise treatment outcome in treatment groups is shown in - Table 3. Relapse was two times higher in FTG as compared to NTG (8.5\% vs. $4.1 \%$ ). Clinical outcome in complicated versus uncomplicated UTI by treatment allocation is depicted in - Table 4. In FTG, relapse and reinfection were seen in all six E. coli isolates. However, among NTG, two Pseudomonas spp., one Citrobacter spp., and one E. coli showed relapse, and reinfection was observed in four E. coli isolates. 
Table 1 Baseline demographics and associated risk factors by treatment allocation

\begin{tabular}{|l|l|l|l|l|}
\hline Variables & Fosfomycin taken, $\boldsymbol{n}=47(\%)$ & Nitrofurantoin taken, $\boldsymbol{n}=96(\%)$ & $\boldsymbol{p}$ - Value \\
\hline \multirow{2}{*}{ Age } & $16-45$ & $19(40.43)$ & $57(59.38)$ & 0.05 \\
\cline { 2 - 5 } & $>45$ & $28(59.57)$ & $39(40.63)$ & 0.000 \\
\hline \multirow{2}{*}{ Sex } & Male & $26(55.32)$ & $19(19.79)$ & $77(80.21)$ \\
\cline { 2 - 5 } & Female & $21(44.68)$ & $13(13.54)$ & 1.00 \\
\hline Associated risk factors & $6(12.77)$ & $3(3.13)$ & 0.147 \\
\hline UTI>2 wk & $5(10.64)$ & $12(12.50)$ & 0.022 \\
\hline Hospitalization > 30 days & $14(29.79)$ & $9(9.38)$ & 0.087 \\
\hline Obstructive uropathy & $10(21.28)$ & $10(10.42)$ & 0.01 \\
\hline Diabetes & $13(27.66)$ & $6(7.79)$ & 0.03 \\
\hline Catheterization & $2(9.52)$ & $18(18.75)$ & 0.002 \\
\hline Pregnancy & $26(55.32)$ & & \\
\hline$>1$ risk factor & & & \\
\hline
\end{tabular}

Abbreviation: UTI, urinary tract infection.

a Percentage calculated from female sex.

Table 2 Organisms isolated and their antimicrobial resistant pattern (\%)

\begin{tabular}{|l|l|l|l|l|l|}
\hline Organism $(n=143)$ & Ciprofloxacin & Cotrimoxazole & Cefazolin & Nitrofurantoin & Fosfomycin \\
\hline Escherichia coli $(n=116)$ & $81(69.3)$ & $78(67.27)$ & $78(67.24)$ & $10(8.62)$ & $0(0)$ \\
\hline Klebsiella spp. $(n=13)$ & $6(46.15)$ & $7(33.8)$ & $7(53.8)$ & $7(53.04)$ & $0(0)$ \\
\hline Pseudomonas spp. $(n=5)$ & $1(20.0)$ & $4(80.0)$ & $3(60.0)$ & $3(60.0)$ & $1(20)$ \\
\hline Citrobacter spp. $(n=3)$ & $0(0)$ & $0(0)$ & $0(0)$ & $2(66.6)$ & $0(0)$ \\
\hline Enterobacter spp. $(n=5)$ & $1(20.0)$ & $3(60.0)$ & $4(80.0)$ & $4(80.0)$ & $0(0)$ \\
\hline Proteus spp. $(n=1)$ & $0(0)$ & $1(100.0)$ & $1(100.0)$ & $1(100)$ & $0(0)$ \\
\hline Total $=143$ & $89(62.2)$ & $93(65)$ & $93(65)$ & $27(18.9)$ & $1(0.69)$ \\
\hline
\end{tabular}

Table 3 Category-wise clinical outcome by treatment allocation

\begin{tabular}{|l|l|l|}
\hline Category & Fosfomycin taken, $\boldsymbol{n}=\mathbf{4 7}(\%)$ & Nitrofurantoin taken, $\boldsymbol{n}=\mathbf{9 6}(\%)$ \\
\hline 1. Sterile urine in follow-up samples & $16(34)$ & $37(38.5)$ \\
\hline $\begin{array}{l}\text { 2. No follow-up sample available but clinical resolution was } \\
\text { confirmed telephonically }\end{array}$ & $22(46.8)$ & $50(53.1)$ \\
\hline $\begin{array}{l}\text { 3. No follow-up sample available but persistence of symptoms } \\
\text { confirmed telephonically }\end{array}$ & $3(6.4)$ & $1(1.04)$ \\
\hline $\begin{array}{l}\text { 4. Reinfection-development of UTI with the different organ- } \\
\text { ism within 30 days }\end{array}$ & $2(4.2)$ & $4(4.1)$ \\
\hline $\begin{array}{l}\text { 5. Relapse-development of UTI with the different organism } \\
\text { within 30 days }\end{array}$ & $4(8.5)$ & $4(4.1)$ \\
\hline
\end{tabular}

Abbreviation: UTI, urinary tract infection.

Table 4 Clinical outcome in complicated versus uncomplicated UTI by treatment allocation

\begin{tabular}{|l|l|l|l|l|}
\hline Classification of UTI & Antibiotic taken & Clinical cure, $\boldsymbol{n}(\%)$ & Clinical failure, $\boldsymbol{n}(\%)$ & $\boldsymbol{p}$-Value \\
\hline \multirow{2}{*}{ Complicated UTI } & Fosfomycin $(n=43)$ & $34(79.1)$ & $09(20.9)$ & 0.192 \\
\cline { 2 - 4 } & Nitrofurantoin $(n=61)$ & $55(91.2)$ & $06(09.8)$ & \multirow{2}{*}{1.000} \\
\hline \multirow{2}{*}{ Uncomplicated UTI } & Fosfomycin $(n=04)$ & $04(100)$ & $0(0)$ & $3(08.6)$ \\
\cline { 2 - 4 } & Nitrofurantoin $(n=35)$ & $32(91.4)$ & & \\
\hline
\end{tabular}

Abbreviation: UTI, urinary tract infection. 


\section{Discussion}

UTI is an exceedingly common type of bacterial infection that affects healthy individual as well as those with comorbidity. Most UTIs are caused by E. coli. ${ }^{1,3,4}$ In the present study, E. coli was isolated in $82 \%$ of cases, which also reflected the etiology of UTI in the general adult population. ${ }^{1,3,4,8}$

There is an increase in literature on the use of fosfomycin in UTIs from the western world, ${ }^{8-11}$ but in India there is limited data on the use of fosfomycin. In the present study, in vitro sensitivity to fosfomycin (99.3\%) was consistent with other studies. ${ }^{1,3}$ Nitrofurantoin is being prescribed in our setup for more than a decade, whereas fosfomycin is being prescribed for the past 2 years. This may be the reason for the higher resistance rate against nitrofurantoin (81.2\%).

Overall, the clinical cure rate was favorable in both treatment groups. Ninety per cent patients receiving nitrofurantoin maintained clinical resolution, versus $80.8 \%$ of those receiving fosfomycin, though this difference was not statistically significant. Our results are comparable with two clinical trials comparing these two drugs; authors of these trials found clinical and microbiological cure rate of 82 to $95 \%$ and 87 to $96 \%$ in FTG and NTG, respectively. ${ }^{9-11}$ Recently, in a randomized clinical trial among women with uncomplicated UTI, 171 of 244 patients (70\%) receiving nitrofurantoin maintained clinical resolution, versus 139 of 241 (58\%) receiving fosfomycin at 24 days of therapy completion. ${ }^{11}$ On the contrary, higher clinical success $(96.4 \%)$ was achieved in the assessment of fosfomycin in complicated UTI. ${ }^{12}$

In an uncontrolled open-label, multicentric study in China, the overall efficacy rate of treatment with fosfomycin in complicated, uncomplicated, and recurrent lower UTI was found to be $64.52 \%, 95.29 \%$, and $77.77 \%$, respectively..$^{13}$ In the present study, the clinical cure rate was observed to be $100 \%$ in uncomplicated UTIs and $80.85 \%$ in complicated UTIs treated with fosfomycin. In NTG, a clinical cure rate of $90 \%$ was maintained in both complicated and uncomplicated UTIs. In another multicenter study from India, the rate of bacterial eradication, bacterial persistence, and bacterial reinfection after fosfomycin treatment was found to be $89.3 \%, 3.9 \%$, and $3.9 \%$, respectively. ${ }^{14}$ In the current study, more than one risk factors were significantly higher in the FTG, which could be the reason for the higher relapse rate in FTG as compared to NTG $(8.5 \%$ vs. $4.1 \%)$.

In the present study, in NTG, 14 patients received this drug, though the 8 isolates were reported resistant and 6 intermediate sensitive to it. In these patients, the drug was prescribed empirically prior to the release of the full susceptibility data from laboratory. Out of these, 12 patients responded to the treatment, as confirmed telephonically. Clinical cure may be due to the synergistic action of the immune system. However, other confounding factors may have contributed for the clinical cure, which requires further study.

National treatment guidelines for antimicrobials have recommended the use of nitrofurantoin, cotrimoxazole, and fluoroquinolone as empiric therapy for lower UTI, but in the current study more than $60 \%$ resistance was observed against cotrimoxazole and fluoroquinolone. Hence, these drugs should not be used as empirical therapy in UTI in the study area. Fosfomycin and nitrofurantoin were found to be the drugs of choice in lower UTIs in outpatients. Patients treated with fosfomycin showed a higher relapse rate as compared to those treated with nitrofurantoin.

\section{Limitations}

This study has important limitations such as uncontrolled nature and single-center experience. Choice of therapy was at the discretion of the treating physician. Same dosage regimen was used for complicated and uncomplicated UTIs. Follow-up urine samples could not be taken in all patients that could have provided a more reliable assessment of the clinical outcome.

\section{Contributions of Authors}

Vinita Rawat conceived, designed, and drafted the manuscript besides analyzing the data. Shradha Sharma performed laboratory tests, data entry, and literature search. Pankaj Kumar Verma critically revised the manuscript and provided samples for testing. Umesh Varshney drafted the manuscript. Rajesh Singh analyzed data and drafted the manuscript.

\section{Conflict of Interest}

Nil.

\section{References}

1 Banerjee S, Sengupta M, Sarker TK. Fosfomycin susceptibility among multidrug-resistant, extended-spectrum -lactamase-producing, carbapenem-resistant uropathogens. Indian J Urol 2017;33(2):149-154

2 Sastry S, Doi Y. Fosfomycin: resurgence of an old companion. J Infect Chemother 2016;22(5):273-280

3 Patwardhan V, Singh S. Fosfomycin for the treatment of drug-resistant urinary tract infections: potential of an old drug not explored fully. Int Urol Nephrol 2017;49(9): 1637-1643 epub ahead of print

4 Mandal J, Acharya NS, Buddhapriya D, Parija SC. Antibiotic resistance pattern among common bacterial uropathogens with a special reference to ciprofloxacin resistant Escherichia coli. Indian J Med Res 2012;136(5):842-849

5 Collee JG, Miles RS, Watt B. Tests for identification of bacteria. In Collee JG, Fraser AG, Marmion BP, Simmons A, eds. Mackie and McCartney's Practical Medical Microbiology, 14th edition. New York:Churchill Livingstone, Elsevier Saunders;2006:135-144.

6 Clinical and Laboratory Standards Institute, Performance Standards for Antimicrobial Susceptibility Testing. 27th Informational Supplement. CLSI Document M100-S27. Wayne, PA: Clinical and Laboratory Standards Institute; 2017

7 Sobel JD, Kaye D. Urinary tract infections. In: Bennett JE, Dolin R, eds. Mandell, Douglas and Benette's Principles and Practice of Infectious Diseases. 8th edition. Philadelphia: Elsevier Saunders;2014:886-913, Chapter 74

8 Matthews PC, Barrett LK, Warren S, et al. Oral fosfomycin for treatment of urinary tract infection: a retrospective cohort study. BMC Infect Dis 2016;16(1):556

9 Stein GE. Comparison of single dose fosfomycin and 7 days of nitrofurantoin in female patients with uncomplicated urinary tract infections. Clin Ther 1992;22:1864-1872 
10 Van Pienbroek E, Hermans J, Kaptein AA, Mulder JD. Fosfomycin trometamol in a single dose versus seven days nitrofurantoin in the treatment of acute uncomplicated urinary tract infections in women. Pharm World Sci 1993;15(6):257-262

11 Huttner A, Kowalczyk A, Turjeman A, et al. Effect of 5 days nitrofurantoin vs single dose fosfomycin on clinical resolution of uncomplicated lower urinary tract infection in women. JAMA 2018;319:1781-1789

12 Giancola SE, Mahoney MV, Hogan MD, Raux BR, McCoy C, Hirsch EB. Assessment of fosfomycin for complicated or multidrug-resistant urinary tract infections: patient characteristics and outcomes. Chemotherapy 2017;62(2):100-104
13 Qiao LD, Zheng B, Chen S, et al. Evaluation of three-dose fosfomycin tromethamine in the treatment of patients with urinary tract infections: an uncontrolled, open-label, multicentre study. BMJ Open 2013;3(12):e00415710.1136/ bmjopen-2013-004157

14 Khawaja AR, Khan FB, Dar TI, Bhat AH, Wani MS, Wazir BS. Fosfomycin tromethamine. Antibiotic of choice in the female patient: a multicenter study. Cent European J Urol 2015;68(3):371-375 